\title{
Review
}

Kale Kundert* and Tanja Kortemme*

\section{Computational design of structured loops for new protein functions}

https://doi.org/10.1515/hsz-2018-0348

Received August 16, 2018; accepted December 18, 2018; previously published online December 20, 2018

\begin{abstract}
The ability to engineer the precise geometries, fine-tuned energetics and subtle dynamics that are characteristic of functional proteins is a major unsolved challenge in the field of computational protein design. In natural proteins, functional sites exhibiting these properties often feature structured loops. However, unlike the elements of secondary structures that comprise idealized protein folds, structured loops have been difficult to design computationally. Addressing this shortcoming in a general way is a necessary first step towards the routine design of protein function. In this perspective, we will describe the progress that has been made on this problem and discuss how recent advances in the field of loop structure prediction can be harnessed and applied to the inverse problem of computational loop design.
\end{abstract}

Keywords: binding site design; loop modeling; positioning functional residues; protein design; Rosetta software.

\section{Introduction: why focus on computational design of structured protein loops}

The routine engineering of functional proteins has been a longstanding goal in the field of computational protein

\footnotetext{
*Corresponding authors: Kale Kundert, Graduate Group in Biophysics, University of California San Francisco, San Francisco, CA 94158, USA; and Department of Bioengineering and Therapeutic Sciences, University of California San Francisco, San Francisco, CA 94158, USA, e-mail: kale@thekunderts.net; and Tanja Kortemme, Graduate Group in Biophysics, University of California San Francisco, San Francisco, CA 94158, USA; Department of Bioengineering and Therapeutic Sciences, University of California San Francisco, San Francisco, CA 94158, USA; and Chan Zuckerberg Biohub, 499 Illinois St, San Francisco, CA 94158, USA, e-mail: tanjakortemme@gmail.com
}

design. However, while the computational engineering of new protein structures has advanced rapidly (Huang et al., 2016), the computational engineering of new functions has been more difficult (Fleishman and Baker, 2012).

One important reason for this discrepancy is that protein structures are largely built from secondary structural elements (e.g. $\alpha$-helices, $\beta$-sheets and canonical turns) with well-understood and predictable patterns of backbone torsion angles and hydrogen bonds, while functional sites (e.g. active sites and binding interfaces) are often built from structured loops with less regular conformations, shaped by the complex and competing requirements of protein function. Early efforts in protein design focused on secondary structure, defining the rules for $\alpha$-helix formation (Errington et al., 2006) and creating simple $\beta$-sheet elements (Lacroix et al., 1999). Exploring the principles of protein secondary structure and their topological arrangements ultimately led to the development of methods - based on the assembly of protein structures from peptide fragments, together with high-resolution sampling methods and allatom energy functions - that have been highly successful in combining helical and sheet elements to create a variety of new, idealized protein folds (Koga et al., 2012).

Now, attention is shifting to the design of protein function. Computational protein design, sometimes in conjunction with directed evolution, has been applied to place catalytic groups (Bolon and Mayo, 2001; Jiang et al., 2008; Rothlisberger et al., 2008; Siegel et al., 2010; Privett et al., 2012), engineer shape-complementary binding interfaces (Chevalier et al., 2002; Kortemme et al., 2004; Fleishman et al., 2011; Karanicolas et al., 2011; Kapp et al., 2012), and switch between different conformational states (Ambroggio and Kuhlman, 2006; Davey et al., 2017). In natural proteins, these functions are more often performed by structured loops than by $\alpha$-helices or $\beta$-sheets, presumably because loops can access a broader range of conformations with greater variation in flexibility or rigidity. For this reason, it seems inevitable that the design of complex protein functions will require the ability to design structured loops with high accuracy. But the same conformational and dynamical breadth that make structured loops functionally useful also makes them challenging 
to design: the number of possible conformations is vast, and even single mutations can have important long-range effects on loop structure and flexibility. Despite these challenges, a few examples of successful loop design have been reported. There have also been significant advances made in the field of loop structure prediction ( $\mathrm{Li}, 2013)$, making it timely to discuss how these advances might be harnessed to computationally design structured loops with greater control than is currently possible.

In this perspective we will begin by discussing examples of functional loops found in nature, to illustrate the different applications that loop design aims to enable. We will then continue by reviewing the progress that has been made to date towards the design of structured loops, before concluding by discussing several promising ways for the field to continue moving forward.

\section{Functional loops in nature}

Many examples of functional loops can be found in enzymes. In fact, loops are much more common in enzyme active sites (50\% of residues) than they are in general (30\% of residues) (Bartlett et al., 2002). One way that loops can contribute to catalysis is by positioning functional groups in the active site (Figure 1A). A good example is ketosteroid isomerase, where the positioning of a general base (Asp38) by a structured loop is estimated to have a 1700fold effect on $k_{\text {cat }}$ (Schwans et al., 2014).

Loops can also contribute to catalysis by acting as a lid for the active site and changing the reaction environment (Figure 1C). For example, upon substrate binding to triose phosphate isomerase (TIM), an active site loop
A

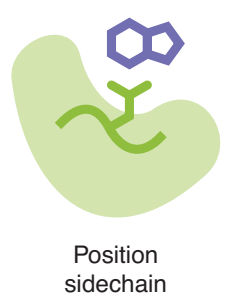

B

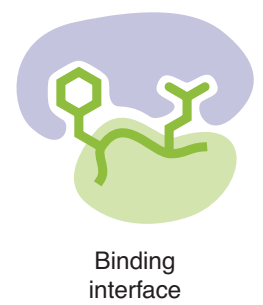

C

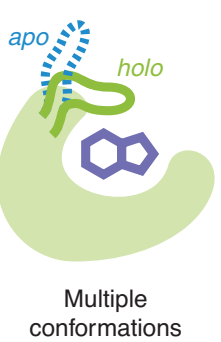

Figure 1: Important applications of loop design. (A) accurately positioning a functional sidechain to interact with a ligand (light green: protein, dark green: loop with functional sidechain, purple: ligand), (B) creating a binding interface (light purple: binding partner), and (C) adopting different functional conformations in response to environmental stimuli, for example, ligand binding (blue, dashed: loop conformation in the absence of ligand, dark green: loop conformation in the presence of ligand). moves more than $7 \AA$ to surround the substrate and hydrogen-bond with the substrate's phosphate group. This substantial conformational change excludes solvent from the active site and prevents the release of reaction intermediates (Pompliano et al., 1990). However, the closed 'lid' also limits the rate of product release, highlighting a carefully balanced trade-off between creating a protected active site environment and exchanging product for substrate. The active site loop structure in TIM is mostly preorganized, moving primarily around a hinge, suggesting that the loop might be optimized to reduce the entropy penalty of closing (Lolis and Petsko, 1990). Rationally designing similar systems will require exquisite finesse.

Structured loops also play an important role in protein-protein interactions (Figure 1B). Perhaps the most prominent examples in this category are antibodies, which use six structured loops - called complementarity determining regions (CDRs) - to bind an astonishing breadth of targets with high affinity and specificity. As antibody CDRs mature, their shape complementarity to their antigen increases (Li et al., 2003; Kuroda and Gray, 2016). Moreover, mature CDRs often adopt conformations that are pre-organized for binding (i.e. conformations that resemble the bound structure, even in the absence of antigen) to minimize the loss of conformational entropy upon antigen binding (Thorpe and Brooks, 2007; Wong et al., 2011; Davenport et al., 2016). However, pre-organization is not a universal feature of high-affinity antibodies (Jeliazkov et al., 2018). Antibodies with less organized CDRs may benefit from the ability to change their conformation to maximize complementarity, or to bind their antigen in multiple modes (James et al., 2003; Wang et al., 2013). The challenge for rational design will be to create loops that can similarly present the complementary surfaces necessary for tight and specific recognition.

Another class of functional loops can be found in proteins that react to their environment. One example is the bacterial outer membrane protein $\mathrm{G}(\mathrm{OmpG})$ that forms a $\mathrm{pH}$-gated pore in the membrane. The gating is mediated by an extracellular strand-loop-strand motif containing two histidine residues (Yildiz et al., 2006; Zhuang et al., 2013). At basic $\mathrm{pH}$, the histidines are neutral and positioned on adjacent strands of the $\beta$-barrel that forms the pore. At acidic $\mathrm{pH}$, protonation of the histidines results in charge repulsion that causes the strands to unfold, lengthening the loop and allowing it to adopt a conformation that covers the pore. Another prominent example is the activation loop present in protein kinases. When phosphorylated, this loop forms contacts that stabilize the active site 
and contributes to catalysis (Steichen et al., 2012). These examples illustrate the utility of being able to design and balance multiple functional loop conformations.

\section{What is loop design?}

Here we define loop design as the problem of predicting sequences that will allow a loop to satisfy certain structural and functional requirements (Figure 2A), such as positioning one or more sidechain groups, adopting a

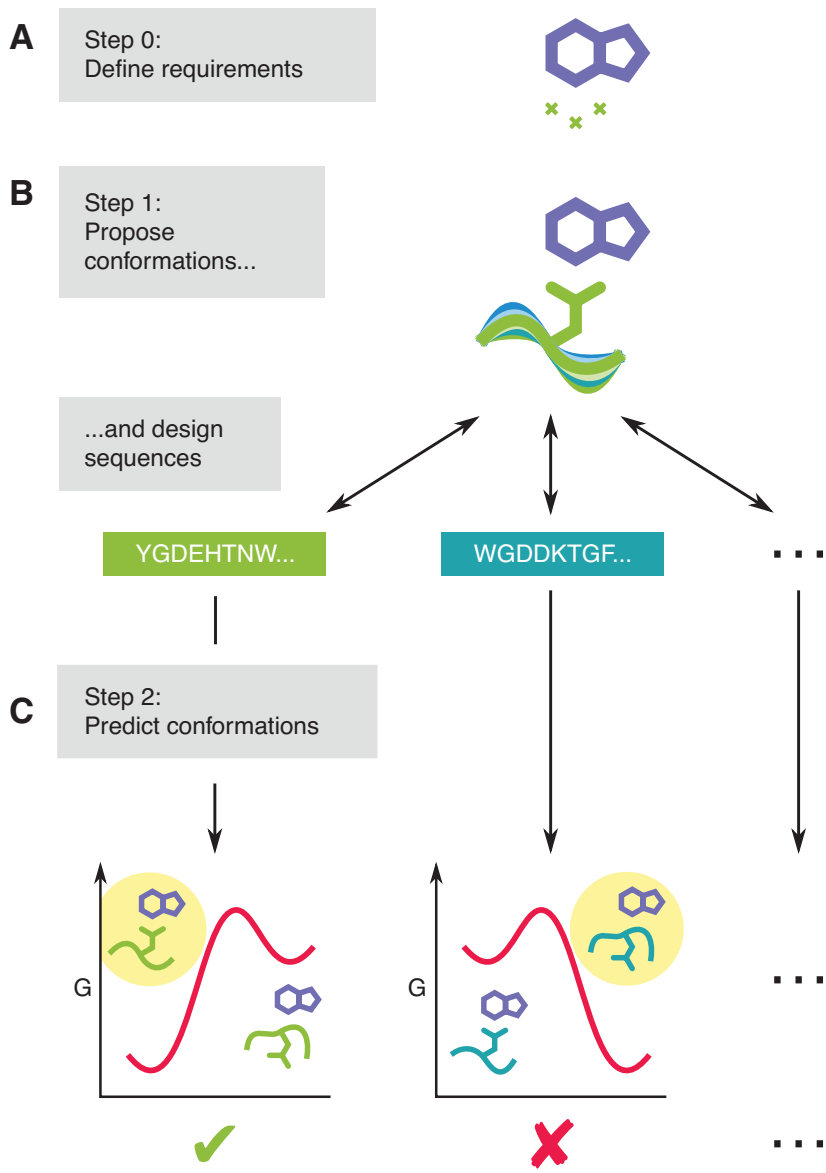

Figure 2: Schematic of a generic loop design protocol. (A) A loop design problem is defined by one or more target interactions (functional requirements). (B) The first step of a generic loop design protocol is to generate design models that satisfy the design goal (shades of blue and green: different design models with different conformations and sequences). (C) The second step of a generic loop design protocol is to identify which models will satisfy the design goal in their minimum free energy conformations. The free energy diagrams each illustrate two hypothetical states, one that satisfies the design goal (left) and one that does not (right). The green checkmark indicates a design that should be experimentally tested, while the red cross indicates a design predicted to be non-functional. particular binding conformation, or changing conformation in the presence of a ligand (Figure 1). We define a loop as a contiguous stretch of protein backbone anchored within a larger scaffold and typically - although not necessarily - lacking secondary structure. For the purpose of this review, we will consider loop design as being distinct from the field of loop grafting, which aims to present a fragment of one protein on the scaffold of another, with important applications in vaccine design (Azoitei et al., 2011; Jardine et al., 2013; Correia et al., 2014). Both loop grafting and loop design aim to create loops in particular conformations. However, for loop grafting the sequence and structure of the loops is known in advance while for loop design determining the sequence and structure of the loops is the key challenge.

It is instructive to consider how a loop design algorithm might operate given a perfect score function and infinite computing resources. In such a hypothetical situation, the first step would be to exhaustively propose design models (combining both sequence and structure) that satisfy the structural requirements without introducing any breaks in the backbone (Figure 2B). The second step would be to subject these designs to intense simulation for the purpose of locating their free energy minima (Figure 2C). Any design that still satisfies the structural requirements in its free energy minimum would be an excellent candidate for experimental validation. In reality, of course, both steps of this algorithm are prohibitively expensive. However, the growing body of literature on loop design (which we will review below) has found various ways to approximate the ideal scenario, for example, by copying fragments of structures from existing proteins, using sophisticated macromolecular structure prediction algorithms, and even incorporating human intuition into the design process.

\section{Loop design: the state of the art}

In spite of the numerous applications, there are not many examples of loop design using computational prediction and design methods. An early example is the effort to improve a monomeric variant of TIM by restabilizing an 8-residue active site loop (Figure 3A) that, in wildtype TIM, participated in the dimer interface (Thanki et al., 1997). In four iterations, computational models of the loop were predicted using Monte Carlo simulations, then mutations were proposed by visual inspection to correct various defects in the models. The final result was a 7-residue loop that improved the activity of monomeric TIM. Furthermore, a 

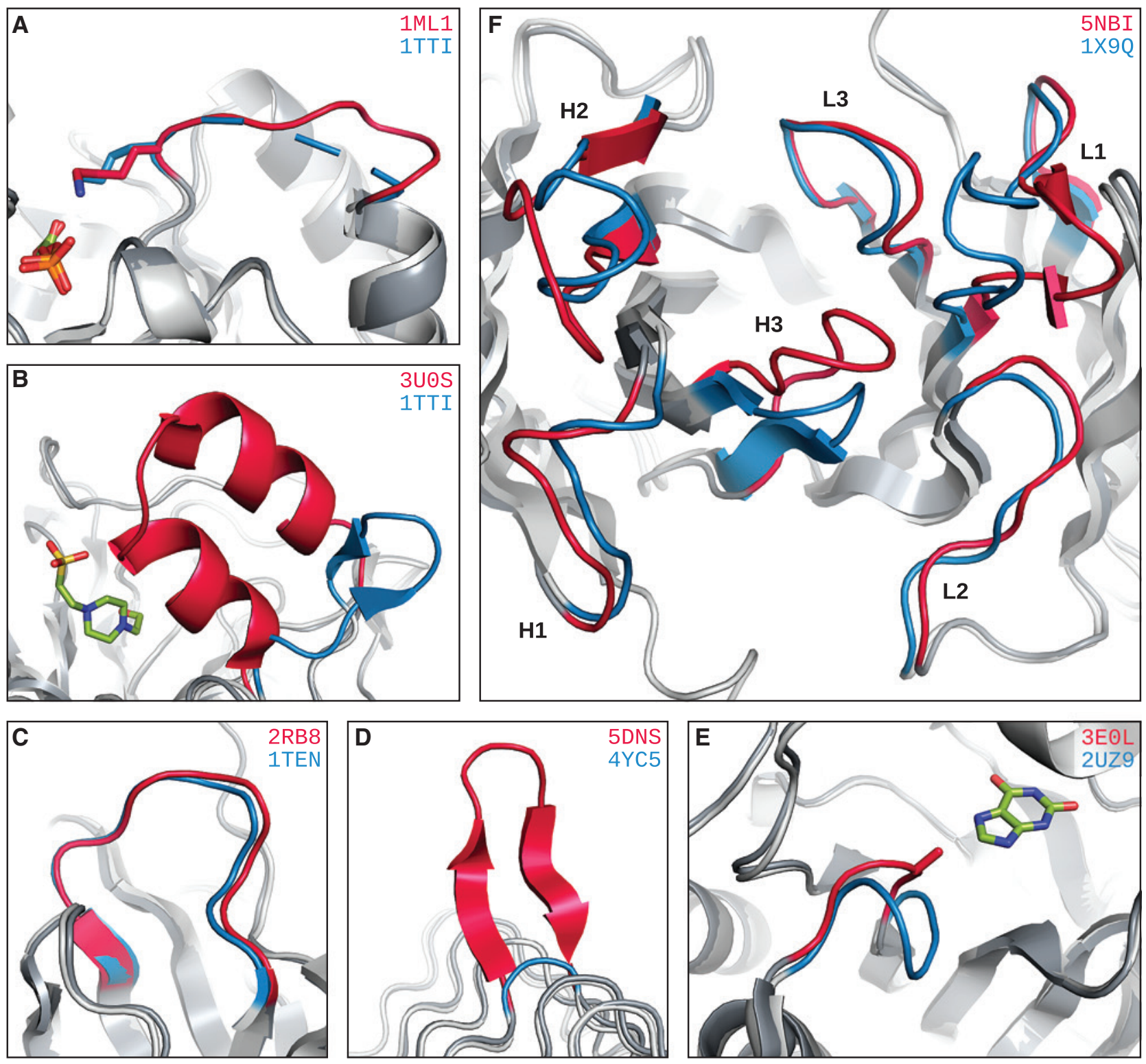

Figure 3: Successful applications of loop design.

Each panel shows crystal structures of a protein with redesigned loops (red and dark gray) and the starting structure (termed scaffold, blue and light gray). The redesigned loops are shown in red and the loops in the starting scaffolds are shown in blue. Ligands (if any are present) are shown in green. PDB IDs for the relevant structures are given in the top-right corner of each panel. Note that the design models are not shown, so these images do not illustrate how accurate the designs were, only how different they were from their starting scaffolds. (A) The stabilized active site loop of MonoTIM (Thanki et al., 1997). The catalytic Lys supported by this loop is shown. The dashed backbone in the scaffold indicates a lack of electron density. (B) The active site of a computationally designed Diels-Alderase (Eiben et al., 2012). (C) An exogenous loop grafted onto the FN3 scaffold (Hu et al., 2007). (D) A de novo loop inserted into a de novo 5-residue-repeat scaffold (MacDonald et al., 2016). (E) The substrate binding loop in the active site of hGDA (Murphy et al., 2009). The C $\beta$ of the Asn intended to bind ammelide is shown, but the remainder of the sidechain was not resolved. (F) The engineered CDR loops (bold labels) of an insulin-binding antibody (Lapidoth et al., 2015). Note that the crystal structure does not include the antigen (insulin).

crystal structure of the designed protein agreed well with the predicted loop conformation ( 0.5 A root mean squared deviation (RMSD) of the backbone heavy atoms $\mathrm{C} / \mathrm{C} \alpha / \mathrm{N} / \mathrm{O}$ in the loop). This report established early on that loop design is both achievable and useful.
Another example of loop design via computational prediction and visual inspection was reported more recently. In this case, players of FoldIt (Cooper et al., 2010) - a gamified version of the Rosetta structure prediction and design programing (Kaufmann et al., 2010; 
Leaver-Fay et al., 2011) - were asked to improve a computationally designed Diels-Alderase (Siegel et al., 2010) by designing an active site loop that would better desolvate the substrate (Eiben et al., 2012). In the first round of the design, the players were allowed to make 5-residue insertions into any of the four active site loops. The authors experimentally tested the four best designs (as judged by the score of the Rosetta energy function and by visual inspection) and over 500 variants of these designs. In the second round of the design, the players were instructed to stabilize the best first-round design through the creation of a helix-turn-helix motif (Figure 3B). This time, the authors tested the two best designs and over 400 variants. The end result was a variant with a 13-residue insertion that improved catalysis by 150 -fold. A model of the final variant created by the players was similar to the crystal structure, except for a rotation in one of the helices (3.1 $\mathrm{C} / \mathrm{C} \alpha / \mathrm{N} / \mathrm{O}$ RMSD). Although the design process required experimentally testing hundreds of variants, it demonstrated that human intuition can guide the design of long and functional loops.

An early example of automated computational loop design was an effort to build new loops into the fibronectin type III (FN3) domain (Hu et al., 2007) (Figure 3C). This domain had already been established as a non-antibody scaffold for evolving loop-based binding interfaces, and like an immunoglobulin domain, it has a $\beta$-sandwich fold from which it presents three mutation-tolerant loops. The authors redesigned one of these loops by searching for 12-residue fragments in the protein data bank (PDB) with similar take-off and landing points to the loops in question (within $3 \AA$ ), grafting each of those fragments onto the FN3 scaffold, repairing the resulting (small) discontinuities in the backbone and finally optimizing the sequence of the inserted residues while allowing slight backbone movement $(\approx 0.3 \AA \mathrm{C} / \mathrm{C} \alpha / \mathrm{N} / \mathrm{O}$ RMSD). Three designs were purified and two were successfully crystallized. One design had the intended loop conformation (0.46 A RMSD), which was similar to the original native loop (0.77 ̊ RMSD). The conformation of the loop in the other design could not be determined due to missing electron density for the loop, which suggests the lack of a single defined conformation. The significance of this work is that it demonstrated that a structured loop could be computationally designed, by borrowing a loop backbone conformation from a naturally existing structure and redesigning the sequence to match the new environment. However, the work did not address the problem of designing function.

A more recent report addressed the design of de novo loops, which were built into a de novo scaffold assembled from 24 repeats of a 5-residue motif (MacDonald et al., 2016) (Figure 3D). The loops were designed by inserting eight residues in the middle of the scaffold, sampling conformations with a coarse-grained and sequenceindependent algorithm, then reconstructing the insertion in full-atom detail and performing fixed-backbone sequence optimization. This protocol produced 4000 loop designs. The conformations represented by these designs (which remained sequence-independent) were assumed to approximate the ensemble of states accessible to an 8-residue loop, allowing the authors to estimate the probability that each design would fold into its intended conformation by threading the design sequence onto each backbone and comparing the resulting Boltzmann-weighted scores. The 10 designs with the highest predicted probabilities of folding correctly were tested. Of these, five could be purified and four could be crystallized. The crystal structures were relatively low-resolution (>3.5 ̊), but two were consistent with their design models, one was inconsistent with its model, and one had missing density for the loop. This report showed that it is possible to create loops with de novo conformations, but these conformations emerged during the design process (rather than being defined a priori) and were not intended to be functional.

Computational loop design was used to alter protein function in an effort to change the substrate specificity of the enzyme human guanine deaminase (hGDA) from guanine to ammelide (Murphy et al., 2009) (Figure 3E). The ultimate goal was to change the substrate specificity of hGDA from guanine to cytosine, but ammelide was chosen as an intermediate step because it resembles guanine on one face and cytosine on the other. The design approach was to remodel the loop in hGDA that positions an arginine (Arg) to recognize guanine to instead position either asparagine (Asn) or glutamine (Gln) with the right geometry to bind the cytosine-resembling face of ammelide. (Interestingly, in natural cytosine deaminases the corresponding Asn/Gln is positioned by a different active site loop, so this project in essence attempted to build a novel active site architecture.) The loop was remodeled by (i) positioning the amide groups of the Asn and Gln sidechains ideally with respect to ammelide, (ii) rotating the sidechain $\chi$ angles to generate backbone conformations capable of supporting that ideal positioning, (iii) superimposing segments from the scaffold on those backbones, (iv) randomly adding or removing residues from either end of those segments, and (v) repairing the backbone with peptide fragment insertions (Simons et al., 1997), cyclic coordinate descent (CCD) (Canutescu and Dunbrack, 2003) and minimization of backbone torsions using Rosetta (Kaufmann et al., 2010). This loop 
remodeling protocol was then followed by fixed-backbone sequence design on the lowest-scoring backbone model (which featured Asn and two deletions) to create designs. A single design (with Gly-Asn-Gly-Val as the loop sequence) was chosen for experimental characterization, based on visual inspection and the results of an unrestrained loop modeling simulation predicting that the design would fold into the desired conformation. The chosen design yielded a 100-fold increase in ammelide deaminase activity, along with a 25 000-fold decrease in guanine deaminase activity. A crystal structure revealed that the loop was close to the computational design model (1.0 $\AA$ C $\alpha$ RMSD), but that the designed Asn was not visible in the electron density. This report is significant because it showed that loops can be designed for function. But there is still room for improvement. The designed loop was relatively short (four residues) and its conformation only differed slightly from that of the starting wildtype structure. For more ambitious design goals, we must learn how to design larger loops and more dramatic conformational changes.

Loop design has also been applied to the problem of computationally designing antibody CDRs to bind particular targets of interest. This is an especially challenging problem for a number of reasons: (i) there are six CDRs, which interact with each other to form a large binding interface, (ii) some of the CDRs, most notably the 3rd CDR on the antibody heavy chain (termed H3), can be long [typically 3-20 residues (Regep et al., 2017)], and (iii) the position and conformation of the antigen must be predicted in concert with the CDRs. However, there is also an exceptional amount of sequence and structural data available for antibodies. These data were recently leveraged to rationally design antibody binding interfaces for human insulin and Mycobacterium tuberculosis acyl-carrier protein 2 (Lapidoth et al., 2015; Baran et al., 2017). The design protocol was premised on the long-standing concept that each CDR except H3 can be assigned to a small number of conformational clusters (Chothia and Lesk, 1987). By combining CDRs from each cluster, 4500 models were created. The epitope was docked against each model, and the antibody sequence was designed to stabilize both the binding interface and the interactions between the CDRs, subject to sequence restraints derived from the natural sequence profiles for each cluster. Each CDR was then optimized by iteratively installing different backbone conformations from the same cluster and re-sampling the sidechains (Lapidoth et al., 2015). With the benefit of manual design and directed evolution, this protocol produced antibodies with mid-nanomolar binding affinities. The anti-insulin antibody was crystallized in its unbound form (Figure 3F) and showed atomic-level accuracy in four of the six CDRs (backbone and sidechain), with the only errors being in H1 and the notoriously difficult H3 (Baran et al., 2017). This method shows that it is possible to design structured loops in binding interfaces, even while also optimizing other degrees of freedom (e.g. epitope docking). The drawback to this method is that it depends on the vast amount of information available for the antibody scaffold. Other common scaffolds, e.g. TIM-barrels might also be amenable to this type of design, but there remains a need for methods that can be applied more generally to any existing scaffold or to new protein folds designed entirely de novo.

\section{What can we learn from loop modeling?}

With the current state of computational loop design in mind, it is interesting and worthwhile to consider how the field might progress in the near future. To do so, it is instructive to examine the related - but much more mature - field of loop modeling. Loop modeling is the problem of predicting the structure of a loop given its sequence. This is the inverse of the loop design problem, which can be framed as predicting sequences that will adopt a particular loop structure. By considering the similarities and differences between these two related problems in the following sections, we will highlight how previous advances in loop modeling can illuminate the way forward in loop design.

The basic structure of a loop modeling algorithm is as follows: The inputs are (i) the sequences of one or more loops and (ii) the atomic coordinates for the remainder of the protein, which might be taken from homology models or experimental structures with missing atoms. The outputs are the atomic coordinates for the loops in question. To produce these coordinates, a loop modeling algorithm has four components: (i) a suitable representation of the system, (ii) an algorithm to sample new loop conformations, (iii) an algorithm to ensure that the protein backbone stays closed, and (iv) an energy function to score different loop conformations. We will discuss each of these components, and how they might be repurposed for loop design, below.

\section{Representation}

There are two main classes of representations employed in loop modeling algorithms: full-atom and coarse-grained. 
Full-atom representations include all protein backbone and sidechain atoms, although most still exclude solvent atoms. Coarse-grained representations strip away some atomic detail in the interest of simplicity. This could mean replacing the sidechain atoms with a single large sphere, removing the sidechain atoms altogether, or retaining only the protein $\alpha$-carbons. One advantage of coarse-grained representations is that they typically have smoother energy landscapes, which can be more thoroughly explored. In contrast, full-atom representations have the potential to be more accurate as details of physical interactions, such as the precise geometries of hydrogen bonds in functional sites, can be modeled. To combine the potential advantages of both classes of representations, many loop modeling methods begin by searching for reasonable loop conformations in a coarsegrained representation, and then switch to a full-atom representation to winnow and refine those conformations (Fiser et al., 2000; de Bakker et al., 2003; Jacobson et al., 2004; Wang et al., 2007; Mandell et al., 2009; Lee et al., 2010). An interesting exception are algorithms that use only a full-atom representation (Das, 2013; Wong et al., 2017). These methods are based on the premise that loops can be sampled stepwise, so these algorithms build loops by sampling each residue in full-atom detail, one-at-atime, until the whole loop has been assembled.

The sequential approach of many loop modeling algorithms - coarse-grained exploration followed by full-atom refinement - may not generally be appropriate for loop design. As already defined, loop design is fundamentally a search for sequences adopting desired conformations subject to functional requirements (Figure 2). Coarsegrained versions of this search could in principle include a representation that encodes a sequence. One such representation is the Rosetta 'centroid-mode', which represents different sidechain types as spheres with different sizes and charge properties (Simons et al., 1997, 1999). However, it is unclear whether sequence-aware coarse-grained representations can encode the functional requirements of a design problem in sufficient detail. A prototypical example is a design goal where functional groups of specific side chains need to be accurately positioned within an active site. In this case, design solutions will need to take into account the specific size and geometry of these sidechains, even during coarse-grained remodeling of the surrounding backbone environment. To address this problem, it will be desirable to develop hybrid representations - perhaps specific to the loop design problem - with tunable levels of detail. For example, one could imagine an algorithm where functional side chain groups are placed using all atom details while the rest of the loop (i.e. the backbone and any peripheral sidechains) is built in lower resolution. Plausible models could then be subjected to full-atom sequence design and structural refinement.

\section{Sampling}

Loop modeling algorithms differ in their approaches to sampling different conformations. These approaches are traditionally categorized as either 'template-based' or 'template-free/de novo' (Shehu and Kavraki, 2012; Li, 2013; Fiser, 2017), where the former query databases of known structures to sample loop conformations, and the latter do not. However, most recent sampling algorithms lie on a continuum between the two. On one side of this continuum are the algorithms that do not borrow three-dimensional coordinates from any existing 'template' protein structure. For example, some algorithms randomly place atoms in a 'cloud' around the loop and subsequently refine them to satisfy certain physical or experimental restraints (Fiser et al., 2000; Liu et al., 2009; Heo et al., 2017). Other algorithms begin with a physically plausible backbone conformation and perturb it via Monte Carlo (Collura et al., 1993; Macdonald et al., 2013) or molecular dynamics (MD) (Rapp and Friesner, 1999; Hornak and Simmerling, 2003; Olson et al., 2011) simulations. A small step along the continuum is to sample backbone torsions from a Ramachandran distribution derived from the frequencies of the $\varphi$ and $\psi$ backbone torsions in high-resolution protein structures (Galaktionov et al., 2001; Xiang et al., 2002; DePristo et al., 2003; Jacobson et al., 2004; Spassov et al., 2008; Mandell et al., 2009; Adhikari et al., 2012; Liang et al., 2014; Tang et al., 2014). This approach has also been extended to two-residue (Stein and Kortemme, 2013) and three-residue (Rata et al., 2010) $\varphi$ and $\psi$ distributions. A further step along the continuum are algorithms that sample new loop conformations by stitching together larger fragments (usually three to nine residues) from known structures (Rohl et al., 2004; Wang et al., 2007; Lee et al., 2010). This fragmentbased approach is based on the assumption that all relevant local conformations are present in the PDB (Simons et al., 1997; Perskie et al., 2008) and is widely recognized for its successful application to the $a b$ initio prediction of protein tertiary structures (Bradley et al., 2005). Finally, on the far side of the continuum are fully template-based algorithms. These algorithms query structural databases for loops of the right length that approximately match the takeoff and landing points of the loop in the input structure (Deane and Blundell, 2001; Michalsky et al., 2003; Fernandez-Fuentes et al., 2006a; Peng and Yang, 2007; 
Choi and Deane, 2010; Holtby et al., 2013; Messih et al., 2015; Marks et al., 2017; Nguyen et al., 2017). Matching loops are usually ranked by how well they fit the gap and align with the input sequence, and can be subsequently relaxed using a full-atom score function. Template-based algorithms can be very fast, a fact that was recently leveraged to create an interactive program for loop modeling and design (Hooper et al., 2018).

In terms of sampling, the clearest difference between loop modeling and loop design is that the former only needs to sample conformation-space, while the latter needs to simultaneously sample conformation- and sequence-space. This poses a challenge called the 'designability' problem (Helling et al., 2001): given a desired conformation, is it possible for some sequence (in some environmental context) to adopt that conformation?

For loop design, one might hypothesize that the template- and fragment-based algorithms (Murphy et al., 2009; Bonet et al., 2014; Lapidoth et al., 2015) might be more successful than de novo methods as the former address the designability problem: if conformations are sampled from a structural database, there is at least one known sequence for each conformation. However, there are still significant challenges in applying templatebased algorithms to the problem of loop design. The most significant challenge is ensuring that the loop will still adopt its conformation in the new structural context of the design. Moreover, template-based methods would need to be modified to account for the additional structural requirements imposed on the loop by the design goal. For example, to design a loop that places the functional group of an active site residue in a defined geometry, a database query would have to find loops that not only start and stop in the right place but are also capable of positioning the residue in question, limiting the number of potential results. This problem is amplified as more residues are included in the design, for example, in large binding sites and protein-protein interfaces. That said, loop design also makes finding suitable loops easier because the algorithm can pick its takeoff and landing points, and the loop can be of any length or sequence. Some design problems can also take advantage of scaffolds belonging to large families with many homologs of known structure, like antibodies or TIM-barrels, for which template-based algorithms are especially likely to be successful. Taken together, it is not clear a priori how difficult it will be to apply template-based algorithms to loop design. However, fragment-based sampling algorithms might be more generally applicable. They offer analogous advantages to the template-based algorithms in terms of designability, but as the backbone can be built by combination of different shorter fragments rather than one large segment, it might be easier to find solutions that accommodate functional requirements imposed by the design goal (which could be expressed using spatial restraints, for example).

Another aspect of sampling in structure prediction is the difficulty of traversing large barriers in the energy landscape, leading to simulations that get trapped in local minima and fail to produce native conformations. A common strategy for addressing this problem is simulated annealing, whereby the temperature of the simulation is gradually increased (to traverse barriers) and decreased over the course of the simulation (Collura et al., 1993; Rapp and Friesner, 1999; Fiser et al., 2000; Rohl et al., 2004; Wang et al., 2007; Mandell et al., 2009; Adhikari et al., 2012; Macdonald et al., 2013; Liang et al., 2014). A related alternative is parallel tempering, whereby simulations at different temperatures are run simultaneously and occasionally swap coordinates (Olson et al., 2008, 2011). Unlike simulated annealing, parallel tempering produces ensembles with defined temperatures. Although such ensembles may be helpful for estimating entropies, few loop modeling applications have applied this strategy as of yet. Genetic algorithms have also been used to enhance sampling (Li et al., 2011; Park et al., 2014; Heo et al., 2017). While genetic algorithms can traverse barriers efficiently, they have to confront the fact that crossover operations involving backbone torsions are likely to produce large clashes (Unger, 2004). Lastly, a handful of methods have attempted to exhaustively sample conformational space, subject to some binning and pruning (Jacobson et al., 2004; Spassov et al., 2008; Das, 2013; Wong et al., 2017).

At least in principle, any of these barrier traversal strategies could be applied to loop design, especially to the step where proposed designs are simulated to assess which will in fact adopt the desired conformation (Figure 2C). This computational 'validation' step (more specifically a consistency check) is similar to a loop modeling simulation, but with a subtle difference: the simulation can stop as soon as it finds a robust number of alternative conformations with lower predicted energies than the design being validated. This difference could allow the validation problem to be recast as a comparison between a small number of plausible off-target states, rather than as a large-scale search of conformational space for the energy minimum. In turn, this comparison could be addressed using enhanced sampling techniques that estimate the free energy difference between small numbers of known states (Kastner, 2011; Comer et al., 2015). 


\section{Closure}

A specific feature of loop sampling algorithms is that they must be able to sample new loop conformations without creating chain breaks in the protein backbone. This problem is referred to as loop closure. A conceptually simple (although combinatorially complex) solution is to allow the sampling algorithm to build the loop from both ends, and to keep the fraction of models that meet in the middle (DePristo et al., 2003; Jacobson et al., 2004; Das, 2013). This approach is common for sampling algorithms that are enumerative. Another solution is to define a score term that favors a closed backbone (e.g. a harmonic restraint across the break) and to let the sampling algorithm (or a gradient minimizer) find conformations that satisfy that term (Collura et al., 1993; Fiser et al., 2000; Rohl et al., 2004; Fernandez-Fuentes et al., 2006b; Spassov et al., 2008; Liu et al., 2009; Adhikari et al., 2012; Macdonald et al., 2013; Tang et al., 2014; Heo et al., 2017). However, this solution may require spending a significant amount of time sampling conformations that are not closed, which is inefficient. An alternative is to use inverse kinematics algorithms borrowed from the field of robotics that calculate the accessible conformations of objects subject to constraints, such as determining the possible positions of the interior joints of a robot arm given fixed positions for the shoulder and fingertips. In the context of loop modeling, such algorithms can be used after sampling to adjust the backbone torsions in the loop such that the loop remains closed. Iterative inverse kinematics algorithms such as cyclic coordinate descent (CCD) (Canutescu and Dunbrack, 2003) converge on a closed backbone over a series of steps and have been applied in many protocols (Shenkin et al., 1987; Xiang et al., 2002; Wang et al., 2007; Minary and Levitt, 2010; Li et al., 2011; Liang et al., 2014; Marks et al., 2017). Analytical inverse kinematics algorithms such as kinematic closure (KIC) (Coutsias et al., 2004) calculate exact solutions to the closure problem using 6 degrees of freedom (such as backbone torsions) to achieve closure, allowing any other degrees of freedom to be sampled freely. These algorithms have also been used in many protocols (Wedemeyer and Scheraga, 1999; Coutsias et al., 2004; Mandell et al., 2009; Lee et al., 2010; Park et al., 2014; Wong et al., 2017). Loop design will require efficient sampling in sequence- and conformation-space, so the efficiency of the inverse kinematics methods makes them good choices for maintaining closure. KIC in particular can simultaneously satisfy multiple geometric restraints [such as ring closure (Coutsias et al., 2016), disulfide bonding
(Bhardwaj et al., 2016) and catalytic group placement], which may be valuable for loop design.

\section{Scoring}

The final component of a loop modeling algorithm is the score function used to evaluate which conformations are the most realistic. Loop modeling algorithms lie on a continuum based on the score function they employ. On one side of the continuum are the algorithms that use physical score functions like AMBER (Rapp and Friesner, 1999), CHARMM (Olson et al., 2008, 2011; Spassov et al., 2008), and OPLS (Jacobson et al., 2004). Some algorithms also use a 'colony' score term that tries to capture the effects of entropy by favoring the models with the most conformationally similar neighbors (Xiang et al., 2002; Fogolari and Tosatto, 2005). On the other side of the continuum are algorithms that use statistical score functions derived from distributions of atoms and residues observed in high-resolution structures, such as DFIRE (Yang and Zhou, 2008; Lee et al., 2010; Holtby et al., 2013; Wong et al., 2017), DOPE (Adhikari et al., 2012), SOAP-Loop (Marks et al., 2017), GOAP (Zhou and Skolnick, 2011) and others (Galaktionov et al., 2001; Macdonald et al., 2013). However, many loop modeling algorithms use hybrid score functions which include both physical and statistical terms (Fiser et al., 2000; de Bakker et al., 2003; Rohl et al., 2004; Wang et al., 2007; Mandell et al., 2009; Li et al., 2011; Liang et al., 2014; Park et al., 2014; Heo et al., 2017). Some methods use a statistical score function for coarse-grained sampling and a physical score function in the full-atom sampling stage.

What considerations are relevant to loop design? Hybrid score functions have been shown to be successful in many applications of computational protein design using Rosetta (Huang et al., 2016). Although there are clear examples of shortcomings (Mandell et al., 2009; Das, 2011; Dou et al., 2017), all of the computational loop design methods reviewed above used the Rosetta score function (Kuhlman et al., 2003; Alford et al., 2017). While other score functions could also be applied to loop design, one consideration is the need for a score term that allows for the comparison of models with different sequences. For example, arginine might be more likely to score better than alanine simply because the former has more atoms, and thus more opportunities to make favorable contacts. Design score functions must use an additional score term (called a 'reference energy' in Rosetta) to counteract this bias.

Another consideration for loop design is the solvent model. While many loop modeling methods use an 
implicit solvent model for computational efficiency, it may be possible to apply explicit solvent models in the context of loop design. As mentioned in the section on barrier traversal, the computational validation step of a loop design protocol (Figure $2 \mathrm{C}$ ) may be able to devote more time towards a small number of structures, allowing the use of more resource-intensive techniques. As loops are typically solvent exposed, an explicit treatment of the solvent may yield worthwhile improvements in accuracy.

\section{What problems are unique to loop design?}

Having discussed loop design in the context of loop modeling, let us now focus on aspects that are specific to loop design. The first of these aspects is a technical consideration: how many residues should be in the designed loop? The loop must be long enough to address the design goal (e.g. if the goal is to position a residue, the loop must be able to reach said residue), but ideally as short as possible. Not only are shorter loops less likely to be conformationally heterogeneous, they are also easier to model accurately. The most naive approach to designing loop length is to simply try several different lengths, but this is inefficient. Loop design already has to grapple with the enormous task of sampling both sequence- and conformation-space. Two more thoughtful approaches have already been explored. Murphy et al. randomly added and removed residues from the loop during design and validated their approach with a loop length recovery benchmark (Murphy et al., 2009). Lapidoth et al. sampled loop sequences and conformations from a database, which included loops of different lengths (Lapidoth et al., 2015). However, neither of these approaches modified their score function to compare loops of different lengths. Just as score functions will prefer large amino acids over short ones (as described already), so too will they prefer long loops over short ones. While this bias did not prevent either group from creating successful designs, it could be addressed using the worm-like chain model to create a reference state for loop lengths. Specialized algorithms are also needed to make length-independent structural comparisons (e.g. for clustering) (Nowak et al., 2016).

The second, more fundamental problem that loop design must confront is: how can the flexibility or rigidity of a loop be accounted for during the design process? For example, one may wish to ensure that a designed loop is adequately rigid, or conversely, to create a loop with defined functional flexibility. Although it is well known that protein native states are best thought of as occupying an ensemble of conformations, only a handful of loop modeling methods have tried to account for the possibility that a loop might not have a single defined conformation (Shehu et al., 2006; Nilmeier et al., 2011; Marks et al., 2018). This consideration may be less important for loop modeling, where the sequence segments being predicted come from natural proteins and are often well-structured, but it is of immediate importance to loop design, where the sequences being predicted were created computationally and disorder could be a common mode of failure. There are many methods for predicting protein flexibility, and while a recent report has begun addressing the issue of designing flexibility by engineering exchange between different sidechain conformations at equilibrium (Davey et al., 2017), to the best of our knowledge these approaches have not yet been applied to the design of loop flexibility. There are two main approaches for predicting flexibility. The first is to generate an ensemble of possible conformations and then to calculate Boltzmann-averaged quantities (like RMSD) over that ensemble (Hilser and Freire, 1996; Shehu et al., 2006, 2007; Benson and Daggett, 2008; Nilmeier et al., 2011). The challenge with this approach is the expense of computing the ensembles and the impossibility of knowing whether all of the relevant states have been sampled. The ensembles must also be generated by a method that obeys detailed balance, which adds complexity. The second approach is to represent the protein as a graph and to infer rigidity from the connectivity of that graph (Jacobs et al., 2001; Pandey et al., 2005; Dobbins et al., 2008; Sarkar, 2017; Bramer and Wei, 2018). Usually the nodes represent atoms or residues, and the edges represent the covalent and non-covalent interactions between those nodes. The challenge with this approach is that it abstracts the details of protein structure and is often more focused on motions at the domain level than at the individual residue level. It is an open question which approach will work best for loop design.

\section{Closing remarks}

In conclusion, we have reviewed the current state of the loop design field and highlighted several promising avenues for progress in the near future: (i) hybrid representations of functional and structural requirements, (ii) template- or fragment-based sampling, (iii) inverse kinematic closure methods, (iv) hybrid score functions that account for sequence- and length-biases and accurately balance polar interactions and solvation, (v) enhanced 
sampling methods for evaluating competing conformations, and (vi) methods that incorporate loop flexibility and rigidity into the design process. The field has had success designing small loops and antibodies and is poised to continue making progress by repurposing and improving existing loop modeling algorithms. Questions such as how to sample loop lengths and how to make a loop either rigid or flexible still need to be grappled with. That said, we believe that many of the technologies enabling the next steps forward are largely in place. Our hope is these steps will lead to methods capable of routinely and accurately designing structured loops. As loops are an integral feature of many functional proteins - including enzymes, binders and switches - such methods will be a boon to the broader and ongoing effort to design functional proteins using computational methods.

Acknowledgments: We would like to thank Matt Jacobson for insightful comments on the manuscript. We would also like to thank Xingjie Pan, Cody Krivacic and Ziyue Wu for the many discussions that have informed our thinking on this topic.

Funding: This work was supported by grants from the US National Institutes of Health (R01-GM110089) and the US National Science Foundation (DBI-1564692). T.K. is a Chan Zuckerberg Biohub Investigator.

\section{References}

Adhikari, A.N., Peng, J., Wilde, M., Xu, J., Freed, K.F., and Sosnick, T.R. (2012). Modeling large regions in proteins: applications to loops, termini, and folding. Protein Sci. 21, 107-121.

Alford, R.F., Leaver-Fay, A., Jeliazkov, J.R., O’Meara, M.J., DiMaio, F.P., Park, H., Shapovalov, M.V., Renfrew, P.D., Mulligan, V.K., Kappel, K., et al. (2017). The Rosetta all-atom energy function for macromolecular modeling and design. J. Chem. Theory Comput. 13, 3031-3048.

Ambroggio, X.I. and Kuhlman, B. (2006). Computational design of a single amino acid sequence that can switch between two distinct protein folds. J. Am. Chem. Soc. 128, 1154-1161.

Azoitei, M.L., Correia, B.E., Ban, Y.E., Carrico, C., Kalyuzhniy, O., Chen, L., Schroeter, A., Huang, P.S., McLellan, J.S., Kwong, P.D., et al. (2011). Computation-guided backbone grafting of a discontinuous motif onto a protein scaffold. Science 334 , 373-376.

Baran, D., Pszolla, M.G., Lapidoth, G.D., Norn, C., Dym, O., Unger, T., Albeck, S., Tyka, M.D., and Fleishman, S.J. (2017). Principles for computational design of binding antibodies. Proc. Natl. Acad. Sci. USA 114, 10900-10905.

Bartlett, G.J., Porter, C.T., Borkakoti, N., and Thornton, J.M. (2002). Analysis of catalytic residues in enzyme active sites. J. Mol. Biol. 324, 105-121.
Benson, N.C. and Daggett, V. (2008). Dynameomics: large-scale assessment of native protein flexibility. Protein Sci. 17, 2038-2050.

Bhardwaj, G., Mulligan, V.K., Bahl, C.D., Gilmore, J.M., Harvey, P.J., Cheneval, O., Buchko, G.W., Pulavarti, S.V., Kaas, Q., Eletsky, A., et al. (2016). Accurate de novo design of hyperstable constrained peptides. Nature 538, 329-335.

Bolon, D.N. and Mayo, S.L. (2001). Enzyme-like proteins by computational design. Proc. Natl. Acad. Sci. USA 98, 14274-14279.

Bonet, J., Segura, J., Planas-Iglesias, J., Oliva, B., and FernandezFuentes, N. (2014). Frag'r'Us: knowledge-based sampling of protein backbone conformations for de novo structure-based protein design. Bioinformatics 30, 1935-1936.

Bradley, P., Misura, K.M., and Baker, D. (2005). Toward high-resolution de novo structure prediction for small proteins. Science 309, 1868-1871.

Bramer, D. and Wei, G.W. (2018). Multiscale weighted colored graphs for protein flexibility and rigidity analysis. J. Chem. Phys. 148, 054103.

Canutescu, A.A. and Dunbrack, R.L., Jr. (2003). Cyclic coordinate descent: A robotics algorithm for protein loop closure. Protein Sci. 12, 963-972.

Chevalier, B.S., Kortemme, T., Chadsey, M.S., Baker, D., Monnat, R.J., and Stoddard, B.L. (2002). Design, activity, and structure of a highly specific artificial endonuclease. Mol. Cell 10, 895-905.

Choi, Y. and Deane, C.M. (2010). FREAD revisited: accurate loop structure prediction using a database search algorithm. Proteins 78, 1431-1440.

Chothia, C. and Lesk, A.M. (1987). Canonical structures for the hypervariable regions of immunoglobulins. J. Mol. Biol. 196, 901-917.

Collura, V., Higo, J., and Garnier, J. (1993). Modeling of protein loops by simulated annealing. Protein Sci. 2, 1502-1510.

Comer, J., Gumbart, J.C., Henin, J., Lelievre, T., Pohorille, A., and Chipot, C. (2015). The adaptive biasing force method: everything you always wanted to know but were afraid to ask. J. Phys. Chem. B 119, 1129-1151.

Cooper, S., Khatib, F., Treuille, A., Barbero, J., Lee, J., Beenen, M., Leaver-Fay, A., Baker, D., Popovic, Z., and Players, F. (2010). Predicting protein structures with a multiplayer online game. Nature 466, 756-760.

Correia, B.E., Bates, J.T., Loomis, R.J., Baneyx, G., Carrico, C., Jardine, J.G., Rupert, P., Correnti, C., Kalyuzhniy, O., Vittal, V., et al. (2014). Proof of principle for epitope-focused vaccine design. Nature 507, 201-206.

Coutsias, E.A., Seok, C., Jacobson, M.P., and Dill, K.A. (2004). A kinematic view of loop closure. J. Comput. Chem. 25, 510-528.

Coutsias, E.A., Lexa, K.W., Wester, M.J., Pollock, S.N., and Jacobson, M.P. (2016). Exhaustive conformational sampling of complex fused ring macrocycles using inverse kinematics. J. Chem. Theory Comput. 12, 4674-4687.

Das, R. (2011). Four small puzzles that Rosetta doesn't solve. PLoS One 6, e20044.

Das, R. (2013). Atomic-accuracy prediction of protein loop structures through an RNA-inspired Ansatz. PLoS One 8, e74830.

Davenport, T.M., Gorman, J., Joyce, M.G., Zhou, T., Soto, C., Guttman, M., Moquin, S., Yang, Y., Zhang, B., Doria-Rose, N.A., et al. (2016). Somatic hypermutation-induced changes in the 
structure and dynamics of HIV-1 broadly neutralizing antibodies. Structure 24, 1346-1357.

Davey, J.A., Damry, A.M., Goto, N.K., and Chica, R.A. (2017). Rational design of proteins that exchange on functional timescales. Nat. Chem. Biol. 13, 1280-1285.

de Bakker, P.I., DePristo, M.A., Burke, D.F., and Blundell, T.L. (2003). $A b$ initio construction of polypeptide fragments: accuracy of loop decoy discrimination by an all-atom statistical potential and the AMBER force field with the generalized born solvation model. Proteins 51, 21-40.

Deane, C.M. and Blundell, T.L. (2001). CODA: a combined algorithm for predicting the structurally variable regions of protein models. Protein Sci. 10, 599-612.

DePristo, M.A., de Bakker, P.I., Lovell, S.C., and Blundell, T.L. (2003). $A b$ initio construction of polypeptide fragments: efficient generation of accurate, representative ensembles. Proteins 51, 41-55.

Dobbins, S.E., Lesk, V.I., and Sternberg, M.J. (2008). Insights into protein flexibility: The relationship between normal modes and conformational change upon protein-protein docking. Proc. Natl. Acad. Sci. USA 105, 10390-10395.

Dou, J., Doyle, L., Greisen Jr., P., Schena, A., Park, H., Johnsson, K., Stoddard, B.L., and Baker, D. (2017). Sampling and energy evaluation challenges in ligand binding protein design. Protein Sci. 26, 2426-2437.

Eiben, C.B., Siegel, J.B., Bale, J.B., Cooper, S., Khatib, F., Shen, B.W., Players, F., Stoddard, B.L., Popovic, Z., and Baker, D. (2012). Increased Diels-Alderase activity through backbone remodeling guided by Foldit players. Nat. Biotechnol. 30, 190-192.

Errington, N., Iqbalsyah, T., and Doig, A.J. (2006). Structure and stability of the alpha-helix: lessons for design. Methods Mol. Biol. 340, 3-26.

Fernandez-Fuentes, N., Oliva, B., and Fiser, A. (2006a). A supersecondary structure library and search algorithm for modeling loops in protein structures. Nucleic Acids Res. 34, 2085-2097.

Fernandez-Fuentes, N., Zhai, J., and Fiser, A. (2006b). ArchPRED: a template based loop structure prediction server. Nucleic Acids Res. 34, W173-W176.

Fiser, A. (2017). Comparative protein structure modelling. In: From Protein Structure to Function with Bioinformatics (Dordrecht: Springer Netherlands), pp. 91-134.

Fiser, A., Do, R.K., and Sali, A. (2000). Modeling of loops in protein structures. Protein Sci. 9, 1753-1773.

Fleishman, S.J. and Baker, D. (2012). Role of the biomolecular energy gap in protein design, structure, and evolution. Cell 149 , 262-273.

Fleishman, S.J., Corn, J.E., Strauch, E.M., Whitehead, T.A., Karanicolas, J., and Baker, D. (2011). Hotspot-centric de novo design of protein binders. J. Mol. Biol. 413, 1047-1062.

Fogolari, F. and Tosatto, S.C. (2005). Application of MM/PBSA colony free energy to loop decoy discrimination: toward correlation between energy and root mean square deviation. Protein Sci. 14, 889-901.

Galaktionov, S., Nikiforovich, G.V., and Marshall, G.R. (2001). $A b$ initio modeling of small, medium, and large loops in proteins. Biopolymers 60, 153-168.

Helling, R., Li, H., Melin, R., Miller, J., Wingreen, N., Zeng, C., and Tang, C. (2001). The designability of protein structures. J. Mol. Graph Model. 19, 157-167.
Heo, S., Lee, J., Joo, K., Shin, H.C., and Lee, J. (2017). Protein loop structure prediction using conformational space annealing. J. Chem. Inform. Model. 57, 1068-1078.

Hilser, V.J. and Freire, E. (1996). Structure-based calculation of the equilibrium folding pathway of proteins. Correlation with hydrogen exchange protection factors. J. Mol. Biol. 262, 756-772.

Holtby, D., Li, S.C., and Li, M. (2013). LoopWeaver: loop modeling by the weighted scaling of verified proteins. J. Comput. Biol. 20, 212-223.

Hooper, W.F., Walcott, B.D., Wang, X., and Bystroff, C. (2018). Fast design of arbitrary length loops in proteins using InteractiveRosetta. BMC Bioinformat. 19, 337.

Hornak, V. and Simmerling, C. (2003). Generation of accurate protein loop conformations through low-barrier molecular dynamics. Proteins 51, 577-590.

Hu, X., Wang, H., Ke, H., and Kuhlman, B. (2007). High-resolution design of a protein loop. Proc. Natl. Acad. Sci. USA 104, 17668-17673.

Huang, P.S., Boyken, S.E., and Baker, D. (2016). The coming of age of de novo protein design. Nature 537, 320-327.

Jacobs, D.J., Rader, A.J., Kuhn, L.A., and Thorpe, M.F. (2001). Protein flexibility predictions using graph theory. Proteins 44, 150-165.

Jacobson, M.P., Pincus, D.L., Rapp, C.S., Day, T.J., Honig, B., Shaw, D.E., and Friesner, R.A. (2004). A hierarchical approach to allatom protein loop prediction. Proteins 55, 351-367.

James, L.C., Roversi, P., and Tawfik, D.S. (2003). Antibody multispecificity mediated by conformational diversity. Science 299 , 1362-1367.

Jardine, J., Julien, J.P., Menis, S., Ota, T., Kalyuzhniy, O., McGuire, A., Sok, D., Huang, P.S., MacPherson, S., Jones, M., et al. (2013). Rational HIV immunogen design to target specific germline B cell receptors. Science 340, 711-716.

Jeliazkov, J.R., Sljoka, A., Kuroda, D., Tsuchimura, N., Katoh, N., Tsumoto, K., and Gray, J.J. (2018). Repertoire analysis of antibody CDR-H3 loops suggests affinity maturation does not typically result in rigidification. Front Immunol. 9, 413.

Jiang, L., Althoff, E.A., Clemente, F.R., Doyle, L., Rothlisberger, D., Zanghellini, A., Gallaher, J.L., Betker, J.L., Tanaka, F., Barbas, C.F., 3rd, et al. (2008). De novo computational design of retroaldol enzymes. Science 319, 1387-1391.

Kapp, G.T., Liu, S., Stein, A., Wong, D.T., Remenyi, A., Yeh, B.J., Fraser, J.S., Taunton, J., Lim, W.A., and Kortemme, T. (2012). Control of protein signaling using a computationally designed GTPase/GEF orthogonal pair. Proc. Natl. Acad. Sci. USA 109, 5277-5282.

Karanicolas, J., Corn, J.E., Chen, I., Joachimiak, L.A., Dym, O., Peck, S.H., Albeck, S., Unger, T., Hu, W., Liu, G., et al. (2011). A de novo protein binding pair by computational design and directed evolution. Mol. Cell. 42, 250-260.

Kastner, J. (2011). Umbrella sampling. Wiley Interdiscip. Rev. Comput. Mol. Sci. 1, 932-942.

Kaufmann, K.W., Lemmon, G.H., Deluca, S.L., Sheehan, J.H., and Meiler, J. (2010). Practically useful: what the Rosetta protein modeling suite can do for you. Biochemistry 49, 2987-2998.

Koga, N., Tatsumi-Koga, R., Liu, G., Xiao, R., Acton, T.B., Montelione, G.T., and Baker, D. (2012). Principles for designing ideal protein structures. Nature 491, 222-227.

Kortemme, T., Joachimiak, L.A., Bullock, A.N., Schuler, A.D., Stoddard, B.L., and Baker, D. (2004). Computational redesign of 
protein-protein interaction specificity. Nat. Struct. Mol. Biol. 11, 371-379.

Kuhlman, B., Dantas, G., Ireton, G.C., Varani, G., Stoddard, B.L., and Baker, D. (2003). Design of a novel globular protein fold with atomic-level accuracy. Science 302, 1364-1368.

Kuroda, D. and Gray, J.J. (2016). Shape complementarity and hydrogen bond preferences in protein-protein interfaces: implications for antibody modeling and protein-protein docking. Bioinformatics 32, 2451-2456.

Lacroix, E., Kortemme, T., Lopez de la Paz, M., and Serrano, L. (1999). The design of linear peptides that fold as monomeric beta-sheet structures. Curr. Opin. Struct. Biol. 9, 487-493.

Lapidoth, G.D., Baran, D., Pszolla, G.M., Norn, C., Alon, A., Tyka, M.D., and Fleishman, S.J. (2015). AbDesign: An algorithm for combinatorial backbone design guided by natural conformations and sequences. Proteins 83, 1385-1406.

Leaver-Fay, A., Tyka, M., Lewis, S.M., Lange, O.F., Thompson, J., Jacak, R., Kaufman, K., Renfrew, P.D., Smith, C.A., Sheffler, W., et al. (2011). ROSETTA3: an object-oriented software suite for the simulation and design of macromolecules. Methods Enzymol. 487, 545-574.

Lee, J., Lee, D., Park, H., Coutsias, E.A., and Seok, C. (2010). Protein loop modeling by using fragment assembly and analytical loop closure. Proteins 78, 3428-3436.

$\mathrm{Li}, \mathrm{Y}$. (2013). Conformational sampling in template-free protein loop structure modeling: an overview. Comput. Struct. Biotechnol. J. 5, e201302003.

Li, Y., Li, H., Yang, F., Smith-Gill, S.J., and Mariuzza, R.A. (2003). $X$-ray snapshots of the maturation of an antibody response to a protein antigen. Nat. Struct. Biol. 10, 482-488.

Li, Y., Rata, I., and Jakobsson, E. (2011). Sampling multiple scoring functions can improve protein loop structure prediction accuracy. J. Chem. Inf. Model. 51, 1656-1666.

Liang, S., Zhang, C., and Zhou, Y. (2014). LEAP: highly accurate prediction of protein loop conformations by integrating coarsegrained sampling and optimized energy scores with all-atom refinement of backbone and side chains. J. Comput. Chem. 35, 335-341.

Liu, P., Zhu, F., Rassokhin, D.N., and Agrafiotis, D.K. (2009). A selforganizing algorithm for modeling protein loops. PLoS Comput. Biol. 5, e1000478.

Lolis, E. and Petsko, G.A. (1990). Crystallographic analysis of the complex between triosephosphate isomerase and 2-phosphoglycolate at $2.5-\AA$ A resolution: implications for catalysis. Biochemistry 29, 6619-6625.

MacDonald, J.T., Kelley, L.A., and Freemont, P.S. (2013). Validating a coarse-grained potential energy function through protein loop modelling. PLoS One 8, e65770.

MacDonald, J.T., Kabasakal, B.V., Godding, D., Kraatz, S., Henderson, L., Barber, J., Freemont, P.S., and Murray, J.W. (2016). Synthetic beta-solenoid proteins with the fragment-free computational design of a $\beta$-hairpin extension. Proc. Natl. Acad. Sci. USA 113, 10346-10351.

Mandell, D.J., Coutsias, E.A., and Kortemme, T. (2009). Subangstrom accuracy in protein loop reconstruction by roboticsinspired conformational sampling. Nat. Methods 6, 551-552.

Marks, C., Nowak, J., Klostermann, S., Georges, G., Dunbar, J., Shi, J., Kelm, S., and Deane, C.M. (2017). Sphinx: merging knowledge-based and $a b$ initio approaches to improve protein loop prediction. Bioinformatics 33, 1346-1353.
Marks, C., Shi, J., and Deane, C.M. (2018). Predicting loop conformational ensembles. Bioinformatics 34, 949-956.

Messih, M.A., Lepore, R., and Tramontano, A. (2015). LoopIng: a template-based tool for predicting the structure of protein loops. Bioinformatics 31, 3767-3772.

Michalsky, E., Goede, A., and Preissner, R. (2003). Loops In Proteins (LIP) - a comprehensive loop database for homology modelling. Protein Eng. 16, 979-985.

Minary, P. and Levitt, M. (2010). Conformational optimization with natural degrees of freedom: a novel stochastic chain closure algorithm. J. Comput. Biol. 17, 993-1010.

Murphy, P.M., Bolduc, J.M., Gallaher, J.L., Stoddard, B.L., and Baker, D. (2009). Alteration of enzyme specificity by computational loop remodeling and design. Proc. Natl. Acad. Sci. USA 106, 9215-9220.

Nguyen, S.P., Li, Z., Xu, D., and Shang, Y. (2017). New deep learning methods for protein loop modeling. IEEE/ACM Trans. Comput. Biol. Bioinform. DOI: 10.1109/TCBB.2017.2784434.

Nilmeier, J., Hua, L., Coutsias, E.A., and Jacobson, M.P. (2011). Assessing protein loop flexibility by hierarchical Monte Carlo sampling. J. Chem. Theory Comput. 7, 1564-1574.

Nowak, J., Baker, T., Georges, G., Kelm, S., Klostermann, S., Shi, J., Sridharan, S., and Deane, C.M. (2016). Length-independent structural similarities enrich the antibody CDR canonical class model. MAbs 8, 751-760.

Olson, M.A., Feig, M., and Brooks, C.L., 3rd. (2008). Prediction of protein loop conformations using multiscale modeling methods with physical energy scoring functions. J. Comput. Chem. 29, 820-831.

Olson, M.A., Chaudhury, S., and Lee, M.S. (2011). Comparison between self-guided Langevin dynamics and molecular dynamics simulations for structure refinement of protein loop conformations. J. Comput. Chem. 32, 3014-3022.

Pandey, B.P., Zhang, C., Yuan, X., Zi, J., and Zhou, Y. (2005). Protein flexibility prediction by an all-atom mean-field statistical theory. Protein Sci. 14, 1772-1777.

Park, H., Lee, G.R., Heo, L., and Seok, C. (2014). Protein loop modeling using a new hybrid energy function and its application to modeling in inaccurate structural environments. PLoS One 9, e113811.

Peng, H.P. and Yang, A.S. (2007). Modeling protein loops with knowledge-based prediction of sequence-structure alignment. Bioinformatics 23, 2836-2842.

Perskie, L.L., Street, T.O., and Rose, G.D. (2008). Structures, basins, and energies: a deconstruction of the Protein Coil Library. Protein Sci. 17, 1151-1161.

Pompliano, D.L., Peyman, A., and Knowles, J.R. (1990). Stabilization of a reaction intermediate as a catalytic device: definition of the functional role of the flexible loop in triosephosphate isomerase. Biochemistry 29, 3186-3194.

Privett, H.K., Kiss, G., Lee, T.M., Blomberg, R., Chica, R.A., Thomas, L.M., Hilvert, D., Houk, K.N., and Mayo, S.L. (2012). Iterative approach to computational enzyme design. Proc. Natl. Acad. Sci. USA 109, 3790-3795.

Rapp, C.S. and Friesner, R.A. (1999). Prediction of loop geometries using a generalized born model of solvation effects. Proteins 35, 173-183.

Rata, I.A., Li, Y., and Jakobsson, E. (2010). Backbone statistical potential from local sequence-structure interactions in protein loops. J. Phys. Chem. B 114, 1859-1869. 
Regep, C., Georges, G., Shi, J., Popovic, B., and Deane, C.M. (2017). The H3 loop of antibodies shows unique structural characteristics. Proteins 85, 1311-1318.

Rohl, C.A., Strauss, C.E., Chivian, D., and Baker, D. (2004). Modeling structurally variable regions in homologous proteins with Rosetta. Proteins 55, 656-677.

Rothlisberger, D., Khersonsky, O., Wollacott, A.M., Jiang, L., DeChancie, J., Betker, J., Gallaher, J.L., Althoff, E.A., Zanghellini, A., Dym, O., et al. (2008). Kemp elimination catalysts by computational enzyme design. Nature 453, 190-195.

Sarkar, R. (2017). Native flexibility of structurally homologous proteins: insights from anisotropic network model. BMC Biophys. $10,1$.

Schwans, J.P., Hanoian, P., Lengerich, B.J., Sunden, F., Gonzalez, A., Tsai, Y., Hammes-Schiffer, S., and Herschlag, D. (2014). Experimental and computational mutagenesis to investigate the positioning of a general base within an enzyme active site. Biochemistry 53, 2541-2555.

Shehu, A. and Kavraki, L.E. (2012). Modeling structures and motions of loops in protein molecules. Entropy 14, 252-290.

Shehu, A., Clementi, C., and Kavraki, L.E. (2006). Modeling protein conformational ensembles: from missing loops to equilibrium fluctuations. Proteins 65, 164-179.

Shehu, A., Clementi, C., and Kavraki, L.E. (2007). Sampling conformation space to model equilibrium fluctuations in proteins. Algorithmica 48, 303-327.

Shenkin, P.S., Yarmush, D.L., Fine, R.M., Wang, H.J., and Levinthal, C. (1987). Predicting antibody hypervariable loop conformation. I. Ensembles of random conformations for ringlike structures. Biopolymers 26, 2053-2085.

Siegel, J.B., Zanghellini, A., Lovick, H.M., Kiss, G., Lambert, A.R., St Clair, J.L., Gallaher, J.L., Hilvert, D., Gelb, M.H., Stoddard, B.L., et al. (2010). Computational design of an enzyme catalyst for a stereoselective bimolecular Diels-Alder reaction. Science 329, 309-313.

Simons, K.T., Kooperberg, C., Huang, E., and Baker, D. (1997). Assembly of protein tertiary structures from fragments with similar local sequences using simulated annealing and Bayesian scoring functions. J. Mol. Biol. 268, 209-225.

Simons, K.T., Ruczinski, I., Kooperberg, C., Fox, B.A., Bystroff, C., and Baker, D. (1999). Improved recognition of native-like protein structures using a combination of sequence-dependent and sequence-independent features of proteins. Proteins 34, 82-95.

Spassov, V.Z., Flook, P.K., and Yan, L. (2008). LOOPER: a molecular mechanics-based algorithm for protein loop prediction. Protein Eng. Des. Sel. 21, 91-100.

Steichen, J.M., Kuchinskas, M., Keshwani, M.M., Yang, J., Adams, J.A., and Taylor, S.S. (2012). Structural basis for the regulation of protein kinase A by activation loop phosphorylation. J. Biol. Chem. 287, 14672-14680.
Stein, A. and Kortemme, T. (2013). Improvements to roboticsinspired conformational sampling in rosetta. PLoS One 8 , e63090.

Tang, K., Zhang, J., and Liang, J. (2014). Fast protein loop sampling and structure prediction using distance-guided sequential chain-growth Monte Carlo method. PLoS Comput. Biol. 10, e1003539.

Thanki, N., Zeelen, J.P., Mathieu, M., Jaenicke, R., Abagyan, R.A., Wierenga, R.K., and Schliebs, W. (1997). Protein engineering with monomeric triosephosphate isomerase (monoTIM): the modelling and structure verification of a seven-residue loop. Protein Eng. 10, 159-167.

Thorpe, I.F. and Brooks, C.L., 3rd. (2007). Molecular evolution of affinity and flexibility in the immune system. Proc. Natl. Acad. Sci. USA 104, 8821-8826.

Unger, R. (2004). The genetic algorithm approach to protein structure prediction. Appl. Evolut. Comput. Chem. 110, 153-175.

Wang, C., Bradley, P., and Baker, D. (2007). Protein-protein docking with backbone flexibility. J. Mol. Biol. 373, 503-519.

Wang, W., Ye, W., Yu, Q., Jiang, C., Zhang, J., Luo, R., and Chen, H.F. (2013). Conformational selection and induced fit in specific antibody and antigen recognition: SPE7 as a case study. J. Phys. Chem. B. 117, 4912-4923.

Wedemeyer, W.J. and Scheraga, H.A. (1999). Exact analytical loop closure in proteins using polynomial equations. J. Comput. Chem. 20, 819-844.

Wong, S.E., Sellers, B.D., and Jacobson, M.P. (2011). Effects of somatic mutations on CDR loop flexibility during affinity maturation. Proteins $79,821-829$.

Wong, S.W.K., Liu, J.S., and Kou, S.C. (2017). Fast de novo discovery of low-energy protein loop conformations. Proteins 85 , 1402-1412.

Xiang, Z., Soto, C.S., and Honig, B. (2002). Evaluating conformational free energies: the colony energy and its application to the problem of loop prediction. Proc. Natl. Acad. Sci. USA 99, 7432-7437.

Yang, Y. and Zhou, Y. (2008). Specific interactions for $a b$ initio folding of protein terminal regions with secondary structures. Proteins 72, 793-803.

Yildiz, O., Vinothkumar, K.R., Goswami, P., and Kuhlbrandt, W. (2006). Structure of the monomeric outer-membrane porin OmpG in the open and closed conformation. EMBO J. 25, 3702-3713.

Zhou, H. and Skolnick, J. (2011). GOAP: a generalized orientationdependent, all-atom statistical potential for protein structure prediction. Biophys. J. 101, 2043-2052.

Zhuang, T., Chisholm, C., Chen, M., and Tamm, L.K. (2013). NMR-based conformational ensembles explain $\mathrm{pH}$-gated opening and closing of OmpG channel. J. Am. Chem. Soc. 135, 15101-15113. 